\title{
Master Transcription Factors Regulate the DNA Methylation Landscape During Hepatocyte Differentiation
}

\author{
Takahiro Suzuki $^{1,2}$, Erina Furuhata ${ }^{1 \dagger}$, Shiori Maeda ${ }^{1 \dagger}$, Mami Kishima ${ }^{1}$, Yurina \\ Miyajima $^{1}$, Yuki Tanaka ${ }^{1,2}$, Joanne Lim $^{1}$, Hajime Nishimura ${ }^{1}$, Yuri Nakanish ${ }^{1}$, Aiko \\ Shojima $^{1,2}$, Harukazu Suzuki ${ }^{1 *}$ \\ ${ }^{1}$ RIKEN Center for Integrated Medical Science (IMS), Laboratory for Cellular Function Conversion Technology, \\ RIKEN Yokohama Campus, 1-7-22 Suehiro-cho, Tsurumi-ku, Yokohama City, Kanagawa 230-0045, Japan \\ ${ }^{2}$ Graduate School of Medical Life Science, Yokohama City University, 1-7-29 Suehiro-cho, Tsurumi-ku, Yokohama \\ City, Kanagawa 230-0045, Japan \\ $\dagger$ These authors contributed equally to this work. \\ *Correspondence: $\underline{\text { harukazu.suzuki@riken.jp }}$
}

Background: Hepatocytes are the dominant cell type of the human liver, with functions in metabolism, detoxification, and in producing secreted proteins. During the process of hepatocyte differentiation, gene regulation and master transcription factors have been extensively investigated, whereas little is known about how the epigenome is regulated, particularly the dynamics of DNA methylation, and the upstream factors that have critical roles.

Results: By examining changes in the transcriptome and the methylome during in vitro hepatocyte differentiation, we identified putative DNA methylation-regulating transcription factors, which are likely involved in DNA demethylation and maintenance of hypo-methylation in a differentiation stage-specific manner. Of these factors, we further reveal that GATA6 induces DNA demethylation together with chromatin activation at a binding-site-specific manner during endoderm differentiation.

Conclusions: These results provide an insight into the spatiotemporal regulatory mechanisms exerted on the DNA methylation landscape by transcription factors, and uncover a new role for transcription factors in early liver development. 


\section{Background}

Hepatocytes, the major parenchymal cells in the liver, are responsible for key liver functions such as metabolism and detoxification. In embryogenesis, the first fate decision to the hepatocyte lineage is the differentiation of primitive streak cells to definitive endoderm (DE) cells, which are a common precursor of endoderm tissues such as liver, pancreas, and gut. Hepatoblasts are hepatic progenitor cells derived from the DE cells, which then differentiate into fetal-like hepatocytes and mature hepatocytes in a stepwise manner. Thus, hepatocytes emerge from pluripotent stem cells through several progenitor cell types.

Several transcription factors (TFs), including c-Jun, and members of the HNF and GATA families are known to play important roles in liver development and hepatocyte differentiation [1-9]. For instance, transcription factor HNF4A is indispensable for specification and early development of the liver [9]. Furthermore, HNF4A is required for the transcriptional activation of genes such as CYP3A4 and CYP2D6, which are crucial for hepatocyte metabolic functions [3,4]. GATA4 and GATA6 are also essential for development of endoderm-derived tissues and cells, including hepatocytes [6-8]. Notably, GATA6 knock-out mice die around E5.5 due to a deficiency of extra-embryonic endoderm development, which can be rescued by tetraploid embryo complementation assays, and indicating that GATA6 is required for liver development and hepatic specification [8,10-12]. Thus, multiple TFs sequentially and coordinately regulate peripheral genes necessary for hepatocyte differentiation.

Gene expression dynamics are regulated not only by the action of transcription factors but also by epigenetic modifications such as DNA methylation. In mammals, most DNA methylation occurs at the cytosines of $\mathrm{CpG}$ dinucleotides, adding a methyl group at the 5-carbon of the cytosine. DNA methylation of gene regulatory regions appears to be associated with silencing of the expression of the downstream gene [13]. Specifically, gene regulatory regions must be demethylated for activation of the downstream gene. Consistent with this, the DNA methylation profile is dramatically altered during embryogenesis and cellular differentiation, with roles in tightly regulating 
expression of downstream genes [14-17]. Indeed, it is reported that DNA methylation plays a crucial role in the expression of numerous liver-specific genes [12,18-24].

Furthermore, expression of CEBP hepatic transcription factors are affected by treatment with the DNA methyltransferase (DNMT) inhibitor, 5-Aza-dC[25]. Interestingly, the DNMT inhibitor facilitates trans-differentiation of adipose tissuederived stem cells or mesenchymal stem cells to hepatocyte-like cells[26-28]. Collectively, these findings show that DNA methylation is a crucial factor for hepatic differentiation.

The gain of DNA methylation is directly achieved by de novo DNMTs [17,2931], and methylation status is maintained during cell divisions by a maintenance DNMT [31-34]. If DNA methylation maintenance does not work properly, the level of DNA methylation declines upon cell proliferation, which is known as passive DNA demethylation[35]. Alternatively, it is plausible that sequential oxidative processes achieve active DNA demethylation by ten-eleven translocation (TET) enzymes [36-40], followed by base-excision repair[40,41]. In addition, the oxidized forms of methylated cytosine (5-hydroxymethyl cytosines ( $5 \mathrm{hmC}), 5$-formyl cytosine (5fC), and 5-carboxy cytosine $(5 \mathrm{caC}))$ are also depleted by passive demethylation mechanisms, because these bases are not recognized by the maintenance DNA methylation mechanism $[42,43]$. Thus, DNA methylation is a balance between gain and loss of methylated bases.

In addition to the mechanisms by which DNA methylation is gained and lost, mechanisms underlying spatiotemporal regulation of DNA methylation are also critical in understanding the overall dynamics of DNA methylation. We and other groups recently reported that some TFs regulate the timing and site-specificity of DNA demethylation [44-50]. We found that RUNX1, an essential transcription factor for hematopoietic development and immune cell functions, induces DNA demethylation by recruiting the TET enzymes and TDG to their binding sites[44]. We have also identified eight novel DNA-demethylating TFs using a screening method we developed[45]. In addition to our findings, other groups have reported DNA-demethylating TFs with roles in several biological processes[46-50]. Thus, a growing body of evidence suggests 
critical roles for TFs in the regulation of DNA methylation. However, the epigenetic roles of TFs specific for hepatocyte differentiation have yet to be identified.

In the present study, we combine TF binding motif (TFBM) overrepresentation analysis for differentially methylated regions [45] with transcriptome analysis. We identify TFs with putative roles in regulating DNA methylation during hepatocyte differentiation by studying in vitro the process of hepatocyte differentiation from human induced pluripotent stem (iPS) cells. Of these TFs, we validate that GATA6 is a master regulator for both DNA demethylation and chromatin activation in the differentiation of the DE. Our data provide significant insights into the regulatory mechanisms shaping the DNA methylation landscape during hepatocyte differentiation.

\section{Results}

\section{DNA methylation dynamics throughout hepatocyte differentiation}

We induced hepatocytes from human iPS cells in vitro and examined the transcriptome by Cap Analysis Gene Expression (CAGE)[51] (Fig. 1A, B). Expression of pluripotent marker genes (POU5F1 and $N A N O G$ ) was considerably downregulated after day 7 of differentiation and undetectable after day 14 (Fig. S1). In contrast, DE markers (SOX17 and FOXA2) and hepatic markers (HNF1B, PPARA, AFP, and PAXO) were upregulated at day 7 and day 14-to-day 28, respectively (Fig. S1). Notably, because $A F P$ is known to be upregulated in immature hepatocytes and to be downregulated in mature hepatocytes, and $P A X 6$ is a maturation marker of hepatocytes, our data confirmed the in vitro differentiation mimics the whole process of in vivo hepatocyte differentiation[52]. Thus, our time-course samples represent day 0 as iPS cells, day 7 as DE, day 14 as hepatoblasts, day 21 as fetal-like hepatocytes, and day 28 as mature hepatocytes, respectively (Fig. 1A, B).

To investigate changes in DNA methylation during hepatocyte differentiation, we performed a methylome analysis of the time-course samples using MethylationEPIC BeadChip (Illumina). Hierarchical clustering showed that iPS cells and DE cells were segregated from the differentiated cells that followed in the time-course, consistent with 
A

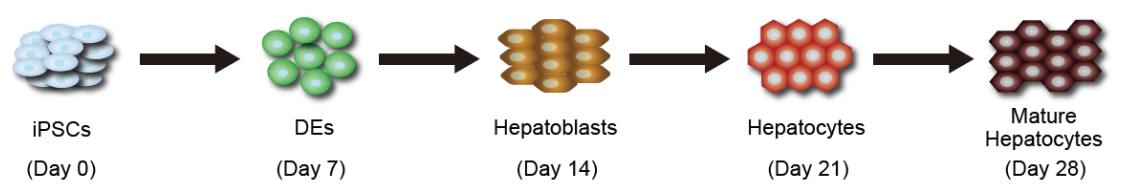

B

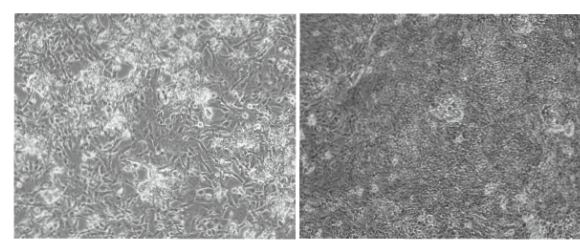

DEs

(Day 7)
Hepatoblasts

(Day 14)

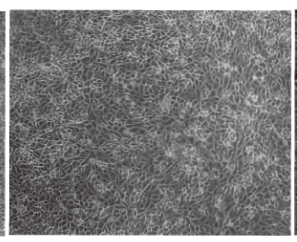

Hepatocytes (Day 21)

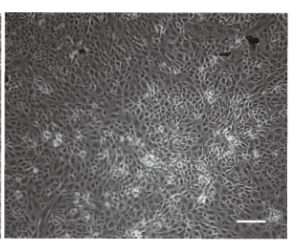

Mature Hepatocytes (Day 28)

C

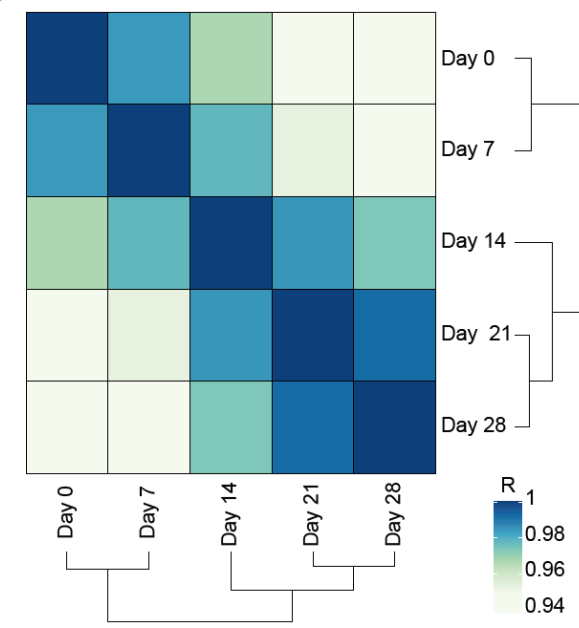

D

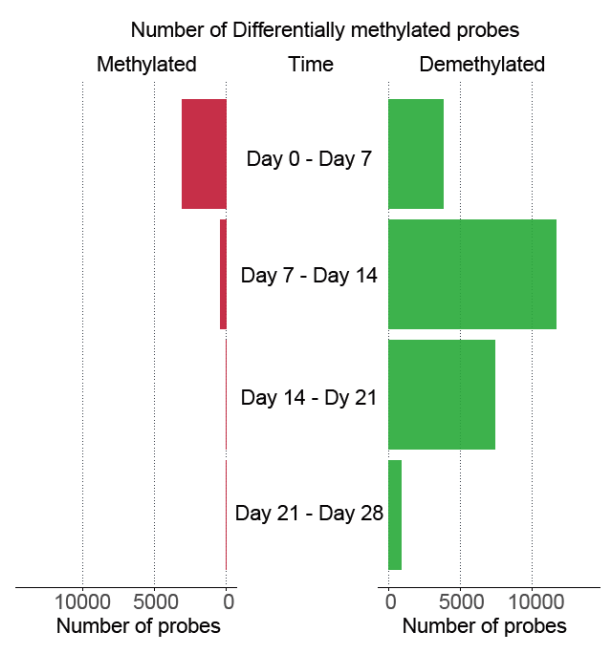

Fig. 1 Time-course methylome analysis of hepatocyte differentiation

(A) Schematic illustration of in vitro hepatocyte differentiation. (B) Pictures of each time point. The scale bar is $200 \mu \mathrm{m}$. (C) A correlation matrix with hierarchical clustering. The color represents the correlation coefficient (R). (D) The number of differentially methylated probes. The left bar plot (red) is methylation and the right bar plot (green) is demethylation.

a commitment to the hepatocyte lineage (Fig. 1C). Comparing adjacent time points, we identified 3088, 446, 38, and 54 methylated CpGs and 3809, 11652, 7383, and 864 demethylated CpGs in each interval (Fig. 1D). Thus, although the gain of methylation mostly occurs in early time points, the number of the differentially methylated CpGs were biased toward demethylation in all intervals, indicating that demethylation (loss of 
methylation) predominates in the dynamics of DNA methylation during hepatocyte differentiation.

We associated biological functions to the differentially methylated regions using the Genomic Region Enrichment of Annotations Tool (GREAT)[53] and summarized the results based on semantic similarity[54]. This analysis revealed an enrichment in development and morphogenesis related Gene Ontologies (GOs), including "pattern specification process", "anatomical structure development", "radial pattern formation", "developmental process", and "regulation of developmental process" (Fig S1B and C). Overall, these results imply that DNA methylation mainly regulates genes related to the developmental process, consistent with specifying the cells into the hepatocyte lineage.

\section{Prediction of DNA methylation-regulating transcription factors throughout hepatocyte differentiation}

We previously developed a screening system to identify TFs which regulate binding site-directed DNA methylation (hereinafter referred to as DNA methylationregulating TFs), which is based on TF binding motif (TFBM) overrepresentation analysis for differentially methylated $\mathrm{CpG}$ regions using ectopic $\mathrm{TF}$ overexpression [45]. By modifying this system, we here performed TFBM overrepresentation analysis for the differentially methylated $\mathrm{CpG}$ regions between two adjacent time points of the differentiation time-course with the TFBM position weight matrix (PWM) database of the IMAGE tool [55]. This database covers most of the known TFs. Because some TFs, such as TFs in the same family, share the same or similar binding motif, the results of TFBM overrepresentation analysis often include false positives. Therefore, to reduce the possibility of false positives, we further narrowed down the overrepresented TFBMs by considering TF expression (CAGE tag per million $(\mathrm{TPM}) \geq 50)$ in either of the two adjacent time points of an interval (Fig. 2A). Thus, by combining methylome and transcriptome analyses, we identified putative DNA methylation-regulating TFs. Comparing each adjacent timepoint, we identified in total 16 putative DNA 


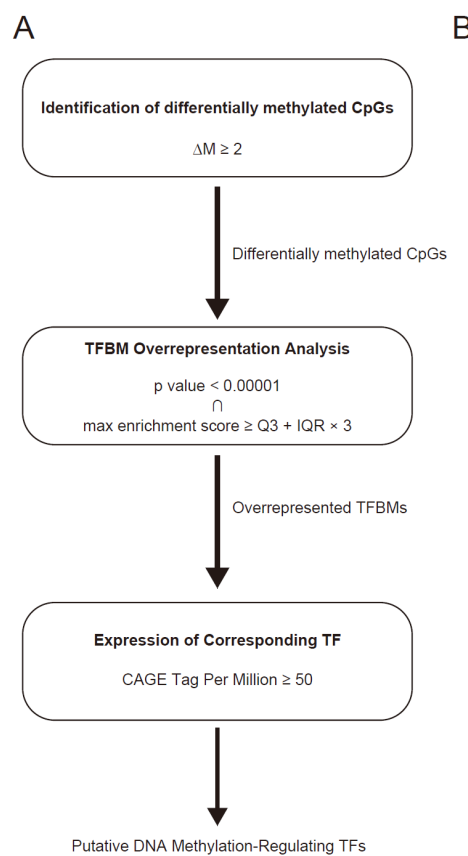

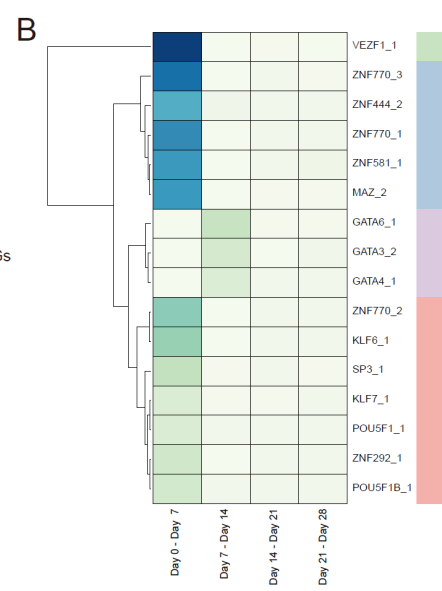
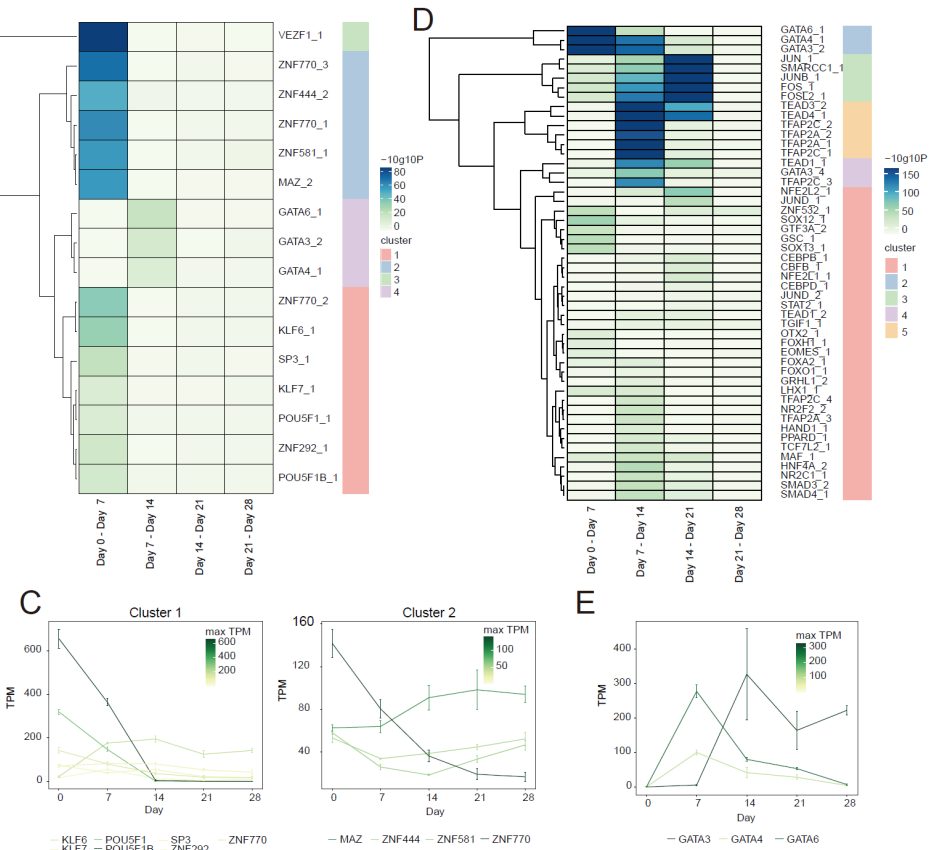

$E$

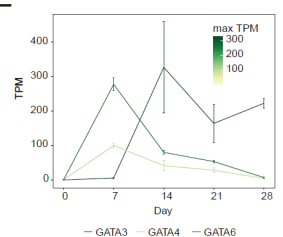

Fig. 2 Prediction of DNA methylation-regulating TFs.

(A) The workflow of DNA methylation-regulating TF prediction. (B, D) Heatmap showing the $p$-value of over-represented TF binding motifs at methylated (B) and demethylated (D) regions. Each column is an interval of adjacent time points. Each row is a putative methylation-regulating TF. Dendrogram of hierarchical clustering is shown at the left of the heatmap and clusters are shown at the right of the heatmap as colors. (C) mRNA expression profile of the cluster 1 and 2 putative DNA methylation-regulating TFs for methylated regions. $X$ - and Y-axes show time points of differentiation (hours from differentiation initiation) and tag-per-million (TPM) of CAGE, respectively. The color of each line represents the maximum TPM. Cluster 3 and 4 and the putative DNA methylation-regulating TFs for demethylated regions were shown in Fig. S2 (E) mRNA expression profile of the GATA3, GATA4, and GATA6. X-and Y-axes show time points of differentiation (hours from differentiation initiation) and tag-per-million (TPM) of CAGE, respectively. The color of each line represents the maximum TPM.

methylation-regulating TFs in the methylated regions. Of these, $13 \mathrm{TFs}$, including POU5F1, a pluripotent cell-specific TF, were identified in the DE differentiation stage (Day 0 -to- Day 7) (Fig. 2B). In addition, GATA6, GATA3, and GATA4 were identified in the hepatoblast differentiation stage (Day 7 -to- Day 14) (Fig. 2B). Interestingly, these putative DNA methylation-regulating TFs for the methylated regions were prone to being highly expressed in the earlier time point of the intervals and then declined along with the progress of differentiation (Fig. 2C and D; Fig. S2A). 
On the other hand, we identified 50 putative DNA methylation-regulating TFs at demethylated regions. Of these, HNF4A, an essential TF for liver development [3][4], was identified in the hepatoblast differentiation stage (Fig. 2D). In addition, the overrepresentation of TFBMs for Activator Protein 1 (AP-1) components such as JUN and FOS, which are involved in the stress response and regeneration in the liver[56-58], increased from the DE differentiation stage to the fetal-like hepatocyte differentiation stage (day 14 -to-day 21) (Fig. 2D). Importantly, GATA6, GATA4, and GATA3, which were also identified in the methylated regions of the hepatoblast differentiation stage, were firstly identified in the DE differentiation stage and overrepresentation of these binding motifs declined as differentiation proceeded (Fig. 2D). Contrary to the putative DNA methylation-regulating TFs for the methylated regions, expression of the putative DNA methylation-regulating TFs for the demethylated regions tends to be upregulated in later timepoints of the intervals (Fig. 2E; Fig. S2B). Taken together, these results suggest that diverse TFs cooperatively regulate the DNA methylation landscape. In particular, GATA transcription factors appear to be the major factors for the DNA methylation regulation, participating in both methylation and demethylation changes.

\section{Ectopic GATA6 overexpression induces binding site-directed DNA demethylation}

Our data suggested the GATA family is a crucial factor for regulating DNA methylation during hepatocyte differentiation, mainly contributing to the demethylation that occurs in DE differentiation. Of the GATA proteins, GATA4 and GATA6 were consistent with the pattern of mRNA expression and are known to be essential TFs for the DE differentiation stage [6-8]. Therefore, we focused the following analysis on possible epigenetic functions of GATA4 and GATA6 in DE differentiation. Firstly, we performed qRT-PCR to confirm the expression changes of GATA4 and GATA6 during the DE differentiation stage. Expression of both GATA4 and GATA6 started increasing from 48 hours after induction of the differentiation and were maximized at 66 hours and 60 hours, respectively, suggesting that GATA6 expression precedes GATA4 expression (Fig. 3A). 
A

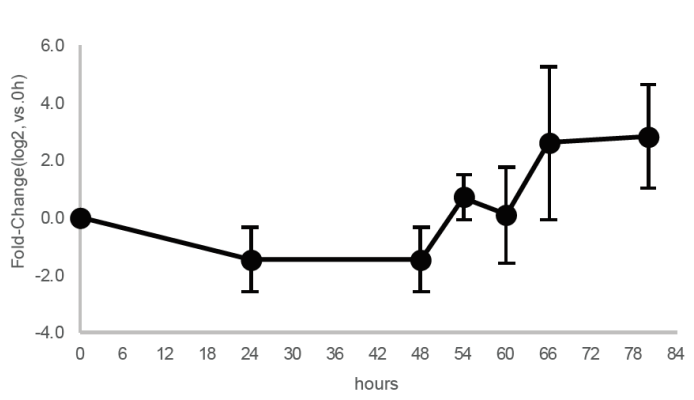

GATA6

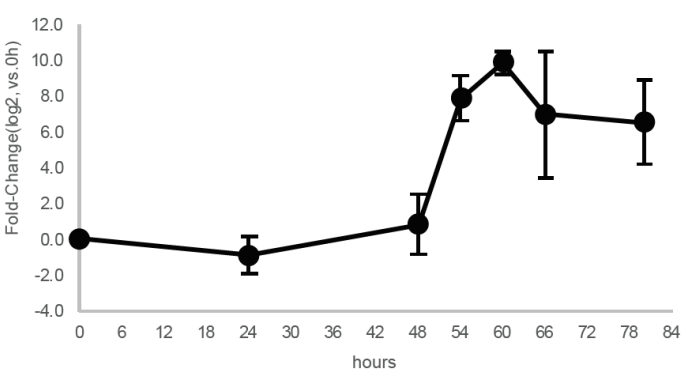

B
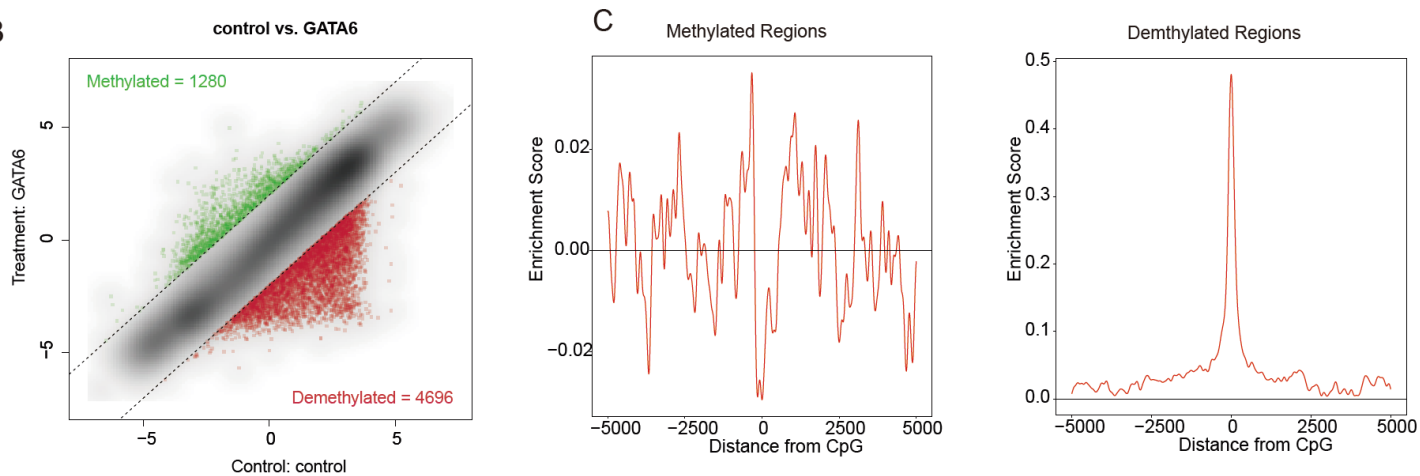

Fig. 3 GATA6-mediated binding site-directed DNA demethylation.

(A) qRT-PCR analysis for GATA4 (left) and GATA6 (right). X- and Y-axes show time points of differentiation (hours from differentiation initiation) and fold-change (compared with 0 hours, $\log _{2}$ scaled), respectively. (B) Scatter plot showing M-value of each probe. X- and Y-axes show M-values of the control sample and GATA6overexpressing sample, respectively. Dotted lines represent $\triangle M=2$ or -2 . Green and red dots are methylated and demethylated probes, respectively, and the number of each probe is shown at the upper left and lower right. (C) Distribution of enrichment score for the GATA6 binding motif within $\pm 5,000$ bp of methylated (left) and demethylated (right) CpG probes in GATA6-overexpressing 293T cells. X-and Y-axes show distance from probe $\mathrm{CpG}$ position and enrichment score, respectively. Horizontal lines are enrichment score $=0$.

Furthermore, GATA6 expression increased drastically, greater than 1,000-fold at 60 hours compared with 48 hours, whereas GATA4 expression increased only 4-fold at 66 hours compared with 48 hours, indicating the dominant impact of GATA6 (Fig. 3A). Indeed, GATA6 is reported to be an upstream factor of GATA4[11]. Therefore, we next overexpressed GATA6 in HEK293T cells, followed by methylome analysis to investigate the role of GATA6 in regulating DNA demethylation. In the HEK293T cells overexpressing GATA6, we identified 1,280 and 4,696 methylated and demethylated CpGs, as compared with mock control transduced cells (Fig. 3B). The motif overrepresentation analysis for the differentially methylated regions revealed that the 
GATA6 binding motif was significantly overrepresented at the demethylated regions but not at methylated regions in the GATA6 overexpressing cells, indicating that GATA6 functions in binding site-directed DNA demethylation (Fig. 3C).

\section{DNA demethylation accompanies GATA6 binding during iPS-DE differentiation}

To investigate the dynamics by which GATA6 regulates DNA demethylation, we performed finer time-course transcriptome and methylome analyses during the timewindow of GATA6 emergence (after 0 hours (h), 48 h, 54 h, 60 h, 66 h, and $72 \mathrm{~h}$ of the differentiation process) (Fig. 4A). T, a marker of the primitive-streak, was upregulated at $48 \mathrm{~h}$ and was downregulated after $54 \mathrm{~h}$. DE markers were upregulated during the period 48 h-to-72 h (Fig. S3A). In agreement with the qRT-PCR analysis (Fig. 3A), the expression of GATA6 was slightly upregulated at $48 \mathrm{~h}$ and drastically increased after 48 $\mathrm{h}$ (Fig. S3A). Hence, our data indicate DE commitment occurs during the period $48 \mathrm{~h}$ to- $72 \mathrm{~h}$ into the differentiation process.

By comparing adjacent time points, we identified 120 (0 h -to- $48 \mathrm{~h}), 94$ (48 h to- $54 \mathrm{~h}), 26$ (54 h -to- $60 \mathrm{~h}), 19$ (60 h -to- $66 \mathrm{~h})$, and 50 (66 h -to- $72 \mathrm{~h})$ methylated CpGs and 220 (0 h -to- 48 h), 226 (48 h -to- 54 h), 33 (54 h -to- 60 h), 27 (60 h -to- 66 h), and 27 (66 h -to- 72 h) demethylated CpGs, respectively (Fig. 4B). However, we did not find the GATA6 binding motif overrepresented at those demethylated regions during any interval (Fig. S3B). Because the time intervals between adjacent time points are 6 hours except for the initial period $(0 \mathrm{~h}$-to- $48 \mathrm{~h})$, the changes in methylation levels may not be enough to be detected as demethylation $(\triangle M>2)$. Indeed, the GATA6 binding motif was overrepresented at the regions demethylated between $0 \mathrm{~h}$ and $72 \mathrm{~h}$ and these demethylated regions tend to be continuously demethylated from $0 \mathrm{~h}$ (Fig. S3C and D). Therefore, to investigate whether the GATA6 binding motif is overrepresented for the cumulative changes in methylation, we compared the regions demethylated at each time point with that at $0 \mathrm{~h}$. We identified 220 ( $0 \mathrm{~h} \mathrm{-to-} 48 \mathrm{~h}), 236(0 \mathrm{~h}$-to- $54 \mathrm{~h}), 416(0 \mathrm{~h}$-to60 h), 876 (0 h -66 h), and 620 (0 h -to- 72 h) demethylated-CpGs (Fig. 4C). Because these demethylated CpGs include those that were demethylated in the earlier time point 
A

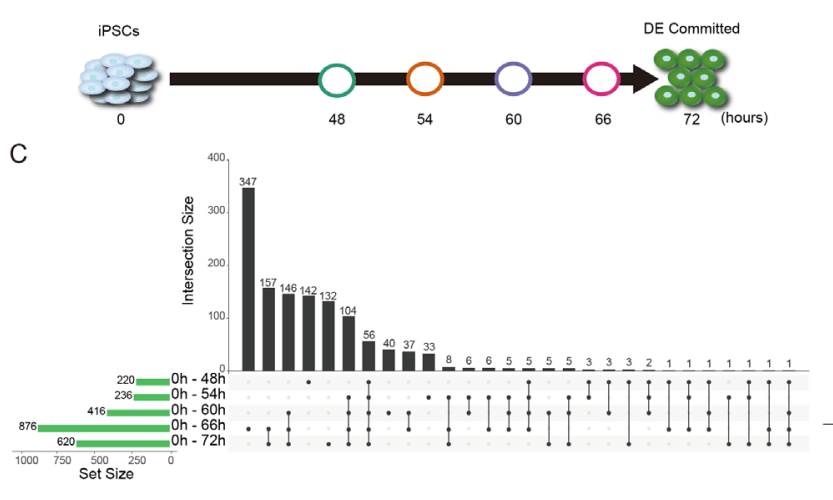

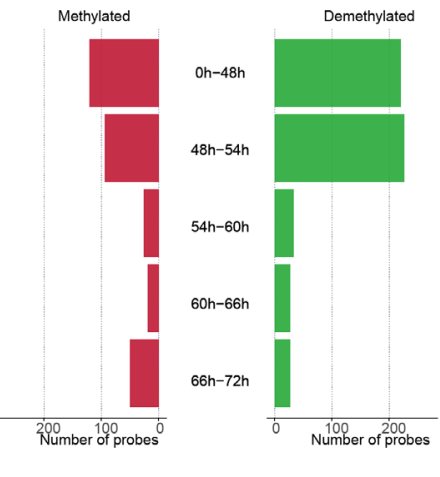
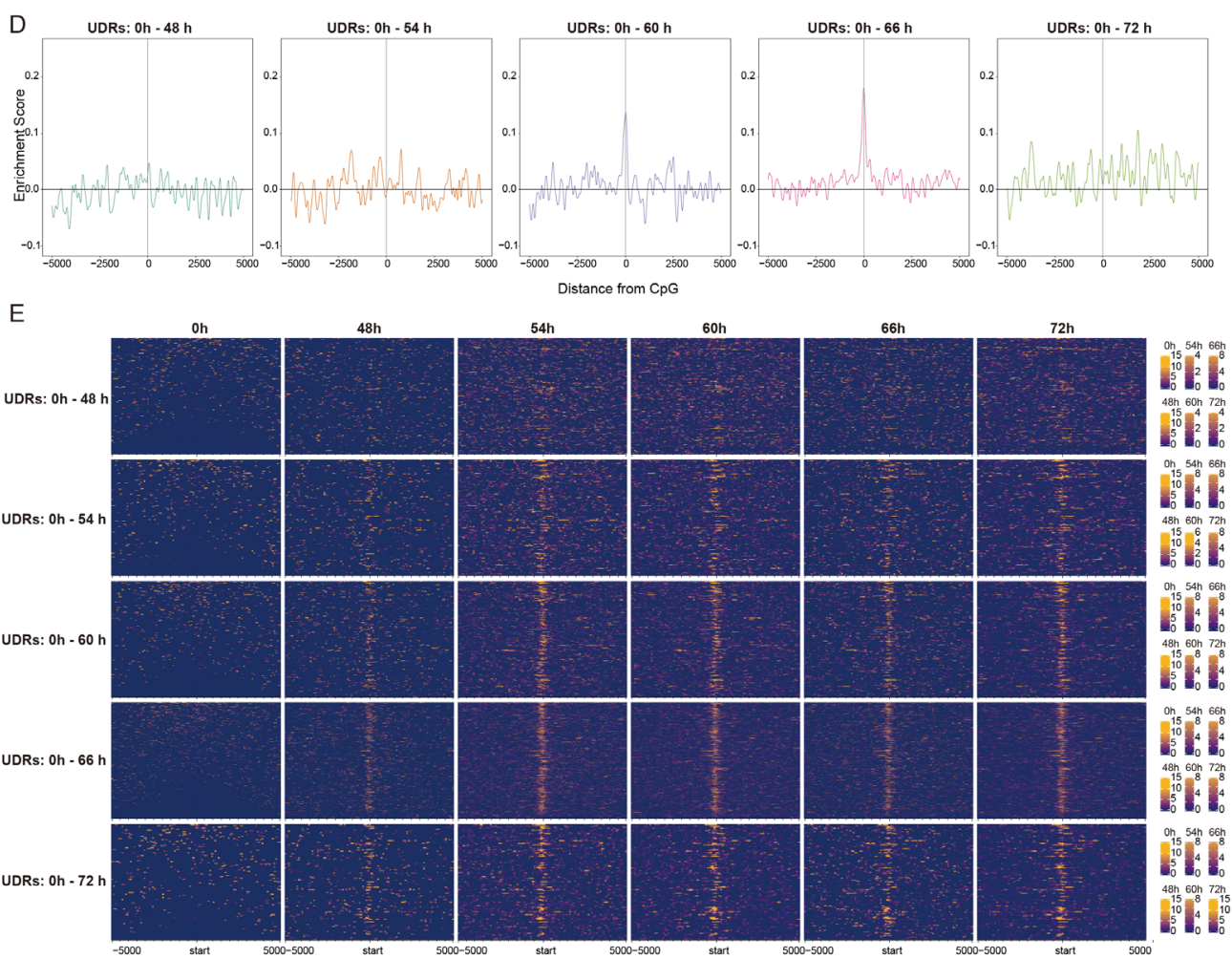

Fig. 4 GATA6-mediated DNA demethylation analysis during DE differentiation (A) Schematic illustration of time-course sampling of DE differentiation. (B) The number of differentially methylated probes. The left bar plot (red) is methylation and the right bar plot (green) is demethylation. (C) UpSet plot showing the demethylated probes at each comparison. The vertical bars indicate the number of intersecting demethylated probes between comparisons, denoted by the connected black circles below the histogram. The horizontal bars show the demethylated probe set size. (D) Distribution of enrichment score for the GATA6 binding motif within $\pm 5,000$ bp of demethylated $\mathrm{CpG}$ probes at each time point compared with undifferentiated iPS cells (0 hours). X-and Y-axes show distance from probe $\mathrm{CpG}$ position and enrichment score, respectively. Horizontal and vertical lines are enrichment score $=0$ and demethylated CpG position, respectively. The colors of each plot represent colors of timepoints shown in Fig. 4A. (E) Enrichment heatmap showing coverage of GATA6 ChIPmentation reads at a range of $\pm 5 \mathrm{kbp}$ from demethylated CpGs. Each time point is horizontally aligned and each of the UDRs are vertically aligned. Dark blue is low coverage and orange is high coverages. 
that were newly detected as demethylated CpGs at each timepoint (referred to as uninherited demethylated CpGs) to clarify the effects of each additional time period. GATA6 motif overrepresentation analysis in the vicinity of these uninherited demethylated $\mathrm{CpG}$ (uninherited demethylated regions: UDRs) revealed the GATA6 binding motif was overrepresented at $0 \mathrm{~h}$-to- $60 \mathrm{~h}$ and $0 \mathrm{~h}$-to- $66 \mathrm{~h}$ (Fig. 4D). To further substantiate the overrepresentation of the GATA6 binding motif at the UDRs, we performed ChIPmentation, which can provide evidence for actual physical interactions between genomic regions and GATA6[59]. Consistent with the expression pattern of GATA6, GATA6 binding was not enriched at UDRs during the period $0 \mathrm{~h}$-to- $48 \mathrm{~h}$, indicating the irrelevance of GATA6 during this period (Fig. 4E). In contrast, unlike binding motif overrepresentation, ChIPmentation showed interactions between GATA6 protein and most of the UDRs of all comparisons apart from the $0 \mathrm{~h}$-to- $48 \mathrm{~h}$, consistent with the expression pattern of the GATA6 (Fig. 4E, Fig. S3A). Because ChIPmentation is more direct evidence of TF binding, we assumed that GATA6 binds to the demethylated regions after $48 \mathrm{~h}$. Thus, our results suggest that GATA6 plays a major role in regulating DNA demethylation during DE differentiation.

\section{The interrelation between DNA demethylation and chromatin status during iPS-DE differentiation}

The majority of the demethylated regions were not promoters but other types of regulatory regions such as enhancers and non-annotated regulatory regions (Fig. S4). Therefore, we investigated the chromatin status of the demethylated regions. Active regulatory regions transcribe several classes of transcripts, including mRNA, promoterupstream transcripts (PROMPTs), and enhancer RNAs (eRNAs), which are typically transcribed within $\pm 250 \mathrm{bp}$ from the center of the regulatory region[60]. Thus, the transcription level serves as an indicator of chromatin activity. Therefore, to investigate the chromatin activity of the demethylated regions, we measured the average TPM of the UDRs ( \pm 250 bp regions from the uninherited demethylated CpGs) by CAGE. The average TPMs of the UDRs were prone to increase as differentiation proceeds in all 

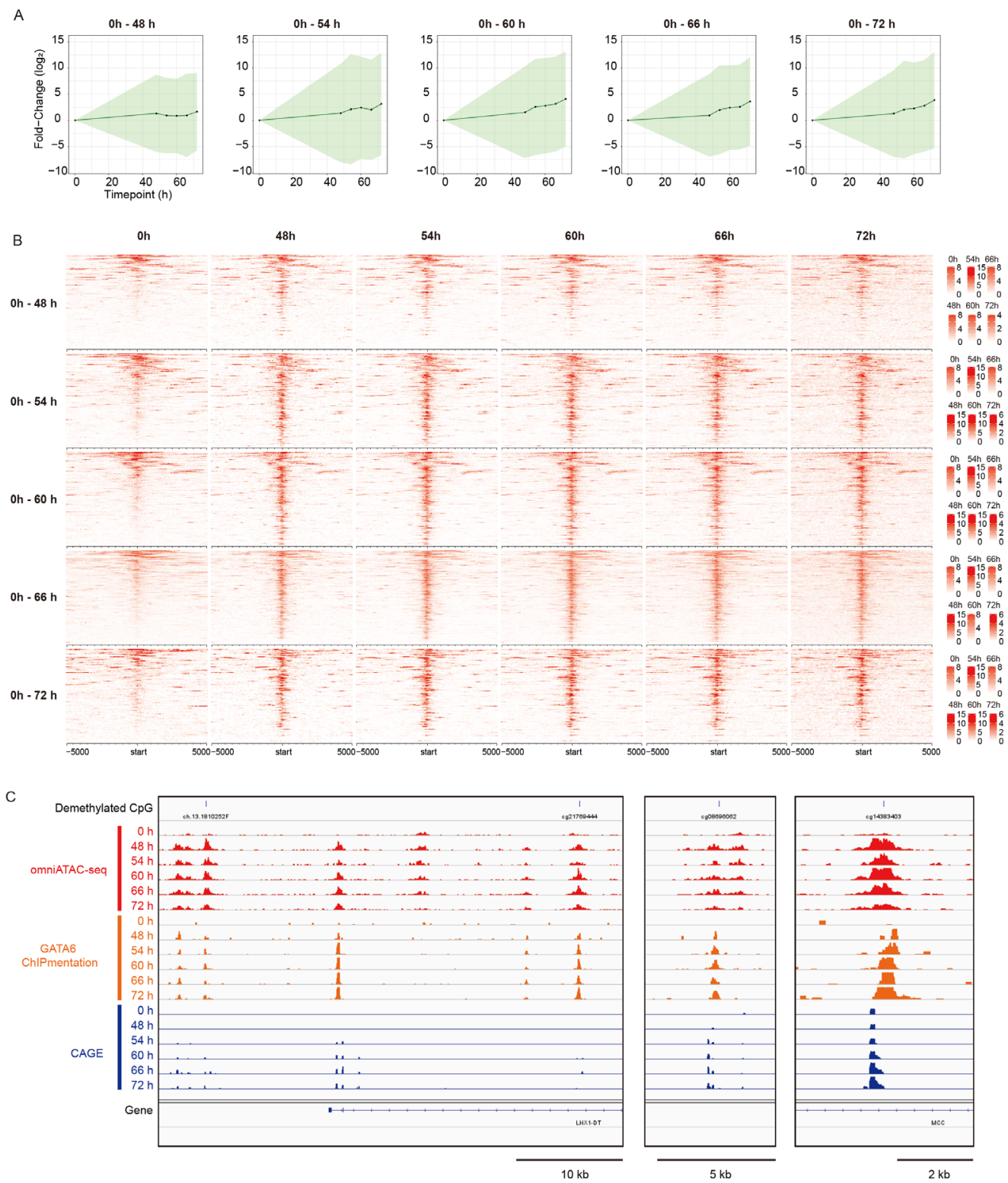

Fig. 5 Chromatin status at demethylated regions.

(A) Change in average TPM of demethylated regions during DE differentiation. $X$ - and Y-axis represents timepoint and relative average TPM (vs. average TPM of $0 \mathrm{~h}$ ), respectively. The light-green shade is the standard deviation. (B) Heatmaps showing Omni-ATAC-seq read coverage at a range of $\pm 5 \mathrm{kbp}$ from demethylated CpGs. Each time point is horizontally aligned and each of the UDRs are vertically aligned. Red is higher coverage of Omni-ATAC-seq reads. (C) A representative screenshot showing DNA demethylated regions, GATA6 ChIPmentation read coverage and OmniATACread coverage.

comparisons except for the $0 \mathrm{~h}$-to- $48 \mathrm{~h}$, indicating the activation of gene regulatory regions (Fig. 5A). 
To further analyze the interrelation between GATA6-mediated DNA demethylation and chromatin status, we measured chromatin accessibility by OmniATAC-seq[61]. Chromatin accessibility at the UDRs increased between $0 \mathrm{~h}$ and $48 \mathrm{~h}$ and was maintained over the following timepoints at most of the demethylated regions (Fig. 5B), in agreement with the transcription pattern and GATA6 binding (Fig. 4E, Fig. S2A, Fig. 5B). Notably, the demethylated regions noted during DE differentiation were only marginally accessible in iPS cells $(0 \mathrm{~h})$, although GATA6 is not expressed at that time, suggesting that target regions of the GATA6-mediated DNA demethylation are pre-defined by chromatin accessibility (Fig. 5B and 5C).

Taking advantage of our time-course multi-omics dataset, we compared the kinetics of GATA6 expression, GATA6 binding to the genome (ChIPmentation), methylation change (M-value), and chromatin status (ATAC-seq and Transcript) (Fig. 6A). Overall, the kinetics of GATA6 binding, chromatin accessibility, and transcription observed the same trends, regardless of the UDRs. Although GATA6 expression was constantly increasing after $48 \mathrm{~h}$, GATA6 binding plateaued at $54 \mathrm{~h}$, although it was somewhat decreased at $66 \mathrm{~h}$. GATA6 transcription levels increased during $0 \mathrm{~h}$-to-48 $\mathrm{h}$ and the expression level was maintained afterward with only slight fluctuations. Of note, chromatin accessibility increased in the period $0 \mathrm{~h}$-to-54 $\mathrm{h}$ and then decreased after peaking, in correlation with the methylation change. Thus, the chromatin activation was achieved before the DNA demethylation occurring during DE differentiation.

\section{Discussion}

In the present study, by applying transcriptome and TFBM overrepresentation analyses for differentially methylated regions, we comprehensively identified putative DNA methylation-regulating TFs active during hepatocyte differentiation. Of these TFs, our results provide multiple strands of evidence that GATA6 is a primary epigenome regulator for the iPSC-to-DE differentiation. 

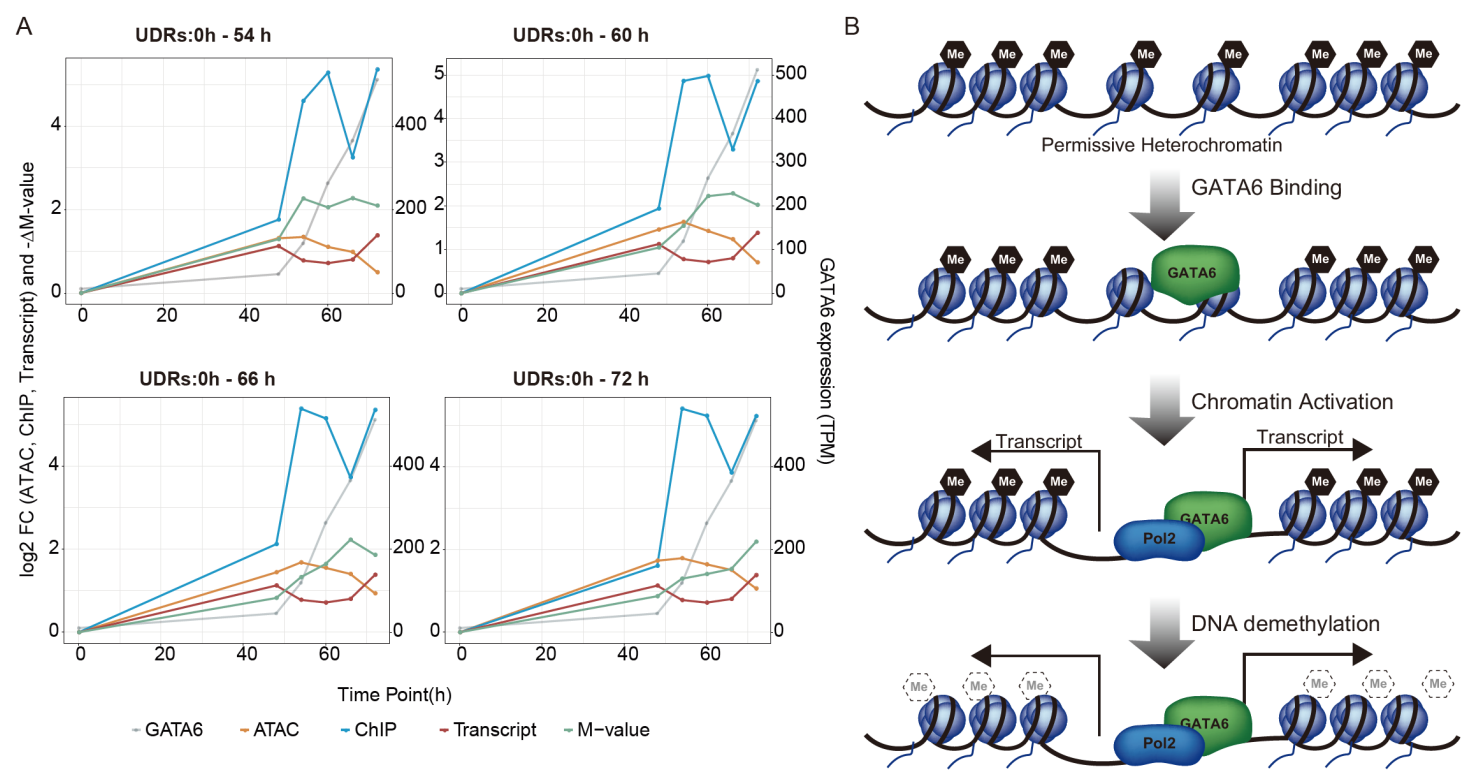

Fig. 6 Multi-omics kinetic analysis

(A) Line plots showing changes in each demethylated region's omics data. X-axis is $\log _{2}$ fold-change (FC) for read coverages of ATAC-seq and ChIPmentation for GATA6 (left scale), and - $\triangle \mathrm{M}$-value (left scale); TPM for GATA6 expression (right scale). Y-axis represents the time points of the differentiation. (B) A schematic illustration showing a model of interrelation between GATA6-mediated DNA demethylation and chromatin status.

Our data suggest that many TFs participate in modulating DNA methylation dynamics in a stage-specific manner. We previously reported that TF-mediated regulation of DNA methylation predominantly manifests as demethylation[45]. Consistent with that report, we found many TFBMs at demethylated regions during hepatocyte differentiation and the methylation change of these TFBMs tended to be correlated with the expression of corresponding TFs. On the other hand, some TFBMs such as POU5F1 (also known as OCT3/4) were overrepresented mainly at the methylated regions during the iPSC-to-DE differentiation and the expression of the corresponding TFs was inversely correlated with methylation change (Fig. 2B and C; Fig. S2A). In comparison, GATA4 and GATA6 showed binding motif overrepresentation at methylated regions of the hepatoblast differentiation stage when GATA4 and GATA6 expression decreases (Fig. 2B and E). Thus, even the gain of methylation may result from the loss of hypo-methylation maintenance by DNA demethylating-TFs. Interestingly, GATA4 and GATA6 binding motifs are also 
overrepresented at demethylated regions of the DE differentiation stage when GATA4 and GAT6 expression increases, showing the dual roles of GATA4 and GATA6 (Fig. 2D and E). To summarize, our data suggest that TF-mediated regulation of DNA methylation acts in both the gain and loss of methylation.

Our data also suggest that HNF4A participates in DNA demethylation during the hepatoblast differentiation stage (Fig. 2D). HNF4A is a crucial TF for hepatocyte differentiation and functions, and is reported to be required during liver development for establishment of 5-hydroxymethyl cytosine $(5 \mathrm{hmC})$ via interactions with TET3 $[3,4,9,62]$. An intermediate modification occurring during DNA demethylation, $5 \mathrm{hmC}$ has a short half-life and is converted to $5 \mathrm{fC}$ and $5 \mathrm{caC}$ by TET proteins[36-41]. Then, $5 \mathrm{fC}$ and $5 \mathrm{caC}$ are rapidly converted to unmodified cytosines by base-excision repair. Because the methylation array analyses used in the present study do not distinguish between methylated cytosine and $5 \mathrm{hmC}$, our results suggest that HNF4A-induced $5 \mathrm{hmC}$ is immediately converted to unmodified cytosine.

Out of the putative DNA-demethylating TFs that we identified, our data demonstrated that GATA6 plays a pivotal role in DNA demethylation during DE differentiation. GATA6 mRNA expression started increasing at $48 \mathrm{~h}$ and was dramatically upregulated during the DE differentiation stage (Fig. 3A and Fig. S2A). In parallel with the expression, binding of GATA6 proteins to the vast majority of demethylated regions was detected and this was maintained through differentiation (Fig. 4E, Fig. 6A), suggesting GATA6 promotes DNA demethylation at its binding sites. In support of this molecular function of GATA6, ectopic expression of GATA6 in HEK 293 T cells proved GATA6-mediated binding site-directed DNA demethylation (Fig. 3C). Thus, these results demonstrate that GATA6 is a crucial regulator of DNA demethylation for early hepatic development, and acts in a binding site-directed manner. 
In the analysis of the iPS cells -to- DE time-course, GATA6 binding motif overrepresentation was not consistent with the results from ChIPmentation for GATA6. Although ChIPmentation showed GATA6 protein binding at the UDRs of all comparisons except $0 \mathrm{~h}$-to- $48 \mathrm{~h}$, binding motif overrepresentation was only detected at the UDRs of the $0 \mathrm{~h}$-to- $60 \mathrm{~h}$ and $0 \mathrm{~h}$-to- $66 \mathrm{~h}$ comparisons. For the GATA6 binding motif overrepresentation analysis, we used the GATA6 PWM of the IMAGE motif database, which includes the canonical GATA binding motif GATW $(\mathrm{W}=\mathrm{A}$ or $\mathrm{T})$. However, GATA-binding proteins can bind various motifs that differ from the canonical GATA-binding motif with comparable affinities [63]. Therefore, TFBM overrepresentation analysis using a known motif database may underestimate the TF binding. Another possibility is that ChIPmentation includes indirect binding of GATA6 via their co-factors. For instance, Friend Of GATA (FOG) proteins, which are co-factors of GATA proteins, have been reported to play essential roles in mediating DNA loop formation[64]. Thus, TFBM overrepresentation analysis with a known motif may not be completely reflecting actual TF binding. Nevertheless, TFBM overrepresentation has a value in predicting the TF binding because it is only based on in-silico analysis without experimental fluctuation. It is also noteworthy that ChIPmentation and ChIP-seq depend highly on the quality of the antibody, which often leads to experimental unreliability.

We also found a relationship between GATA6-mediated DNA demethylation and chromatin activation. Notably, chromatin accessibilities of GATA6 binding regions are already slightly accessible in iPS cells (Fig. 4E), although GATA6 is not expressed at that stage. GATA6 appears to be a pioneer factor that directly binds to permissive heterochromatin and primes the opening of chromatin [65-69]. Consistent with this, our result indicates that the targets of GATA6-mediated DNA demethylation are preliminarily marked by marginal chromatin accessibility. Thus, the chromatin accessibility assay preliminarily indicates the target regions for TF-mediated regulation of methylation. 
Further multi-omic kinetics analysis suggested the temporal relationships that exist between GATA6-mediated DNA demethylation and chromatin activity. Interestingly, chromatin accessibility increased from $0 \mathrm{~h}$-to- $54 \mathrm{~h}$ and then declined afterward, although DNA methylation decreased. This is inconsistent with the notion that DNA methylation is correlated with closed chromatin. The methyl-group of methylated cytosine lies in the major groove of the DNA double helix, which hinders the interaction of TFs with DNA. On the other hand, DNA demethylation increases the affinity of the TFs for their binding site. Therefore, the decrease in chromatin accessibility may be due to occupation of the opened chromatin by TFs. In fact, the chromatin accessibility assay reflects not only the presence of open chromatin or nucleosome density but also TF binding[70]. Thus, our results indicate that the chromatin accessibility assay may not correctly reflect the chromatin activity.

Although the underlying molecular mechanisms have not been investigated in this study, our analysis proposes a sequential reaction takes place, coordinated with the expression pattern of TFs. DNA-demethylating TFs firstly bind to the permissive heterochromatin sites where the TFBM are located. They then open and activate the chromatin at the binding sites, and finally complete DNA demethylation (Fig. 6B). This sequential reaction may be due to differences in reaction times between chromatin remodeling and DNA demethylation, because the level of DNA methylation progressively decreases from the beginning of the differentiation process. While chromatin remodeling is an enzymatic reaction, DNA demethylation is achieved by several mechanisms, including passive DNA demethylation, which depends on cell division. Cell division is a complex process composed of multiple steps, and taking more time than a single enzymatic reaction. Therefore, even if timing for the initiation step is the same, the total reaction time to completion may differ between chromatin remodeling and DNA demethylation. 
In addition to GATA6 having an essential role in physiological endoderm cell development, GATA6 haploinsufficiency causes several diseases such as neonatal diabetes mellitus, cardiomyopathy, and pancreatic agenesis[71-73]. In the present study, we found a novel function of GATA6, regulating binding site-directed DNA demethylation. Hence, epigenetic abnormalities may also be associated with the pathology of these diseases already linked to GATA6. Hence, epigenetic analyses of these diseases deserves to be a priority and may provide novel insights into underlining molecular mechanisms.

\section{Conclusions}

We identified multiple putative DNA methylation-regulating TFs acting at distinct stages throughout hepatocyte differentiation, which are likely involved in DNA demethylation and maintenance of hypo-methylation. Our data suggest that multiple TFs cooperatively modulate the DNA methylation landscape during cellular differentiation. A finer scale analysis of the time-course throughout DE differentiation showed the crucial role of GATA6-mediated DNA methylation regulation, which is gradually completed upon the rapid activation of chromatin.

\section{Methods}

\section{Cell culture and in vitro differentiation}

The 201B7 human iPS cell line was acquired from the RIKEN BioResource Center (BRC) and was cultured in a Cellartis ${ }^{\circledR}$ DEF-CS ${ }^{\mathrm{TM}}$ Culture System (Takara Bio Inc., Shiga, Japan). For in vitro hepatocyte differentiation and DE differentiation, we used the Cellartis ${ }^{\circledR}$ Hepatocyte Differentiation Kit (Takara Bio Inc.) and the Cellartis ${ }^{\circledR}$ DE Differentiation Kit (Takara Bio Inc.), respectively, according to the manufacturers' instructions.

\section{Methylation array analysis}

Genomic DNA was isolated using a NucleoSpin ${ }^{\circledR}$ Tissue Kit (Macherey-Nagel, Düren, Germany). The methylation array used an Infinium Human methylationEPIC BeadChip 
(Illumina, San Diego, CA), according to the manufacturer's instructions. Data was processed as previously described.

\section{Cap Analysis Gene Expression}

Total RNA was extracted using NucleoSpin ${ }^{\circledR}$ RNA (Macherey-Nagel). CAGE libraries were prepared as previously described. Briefly, $3 \mu \mathrm{g}$ of total RNA from each sample were used in reverse transcription reactions with random primers. The $5^{\prime}$ end cap structure was biotinylated and captured with streptavidin-coated magnetic beads (Thermo Fisher). After ligation of 5' and 3' adaptors, second-strand cDNA was synthesized, followed by digestion with exonuclease I (New England BioLabs). The purified CAGE libraries were sequenced using single-end reads of $50 \mathrm{bp}$ on the Illumina HiSeq 2500 (Illumina, USA). The extracted CAGE tags were then mapped to the human hg19 genome by STAR. The tags per million (TPM) were calculated for each FANTOM5 TSS peak and regions extended $\pm 250 \mathrm{bp}$ from each differentially methylated $\mathrm{CpG}$. Gene expression levels of each gene were computed as the sum of multiple TSS peaks associated with a single gene.

\section{Omni-ATAC-seq}

Omni-ATAC-seq libraries were prepared as previously described[61]. Briefly, $5 \times 10^{4}$ cells were stored at $-80^{\circ} \mathrm{C}$ in STEM CELLBANKER ${ }^{\circledR}$ (Takara Bio Inc.) until use. The cells were washed with PBS and nuclei were extracted. The extracted nuclei were resuspended in $50 \mu \mathrm{l}$ of transposition mix (100 nM TED1 (Illumina), $0.01 \%$ digitonin, and $0.1 \%$ Tween-20, in TD buffer (Illumina)) and incubated at $37^{\circ} \mathrm{C}$ for 30 min with 1,000 RPM mixing. DNA was extracted from the reaction mixture with Zymo DNA Clean and Concentrator (Zymo Research, CA, USA). DNA library was prepared using NEBNext ${ }^{\circledR}$ Ultra $^{\mathrm{TM}}$ DNA Library Prep Kit for Illumina ${ }^{\circledR}$ (New England BioLabs) with 5cycles of pre-amplification and 3 to 7 cycles of PCR amplification. Amplified DNA library was purified with Zymo DNA Clean and Concentrator (Zymo Research), followed by two size-selection steps with SPRIselect (1:0.6 and 1:0.2 sample vol. to beads vol.; Beckman Coulter, CA, USA). The libraries' size distribution was determined by Bioanalyzer (Agilent Technologies, CA, USA), and the concentration of the libraries 
was quantified by GenNext NGS Library Quantification Kit (Toyobo Co., Ltd., Osaka, Japan). The Omni-ATAC-seq libraries were sequenced using 150 bp paired-end reads on the HiSeq X (Illumina). The obtained sequence reads were mapped to the human hg19 genome by bowtie2.

\section{Lentivirus preparation and transduction}

GATA6 and ORF were sub-cloned into the CSII-EF-RfA-IRES2-puro vectors using the Gateway LR reaction (Thermo Fisher Scientific Inc.). GATA6 lentivirus vectors were produced by using the LV-MAX Lentiviral Production System (Thermo Fisher Scientific Inc.) according to the manufacturer's instructions. The resulting lentivirus vectors were transduced to $293 \mathrm{~T}$ cells, as described previously.

\section{Quantitative reverse transcription PCR ( $q R T-P C R)$}

qRT-PCR was performed as previously described[45] with primers shown in table S1.

\section{ChIPmentation}

ChIPmentation was performed using a ChIPmentation for Transcription Factor kit (Diagenode) according to the manufacturer's instructions. Briefly, the cells were collected and fixed with $1 \%$ formaldehyde for 8 minutes at RT. The fixed cells were lysed, and chromatin was sheared by sonication using a Picoruptor ${ }^{\circledR}$ (Diagenode) for 10 cycles. The sheared chromatin derived from one million cells was subjected to magnetic immunoprecipitation and tagmentation using an SX-8G IP-STAR ${ }^{\circledR}$ Compact Automated System (Diagenode) with the anti-GATA6 antibody (D61E4, Cell Signaling Technology, Inc.). The immunoprecipitated samples were stripped from the magnetic beads and subjected to end repair and reverse cross-linking. The Illumina sequence compatible sequencing libraries were amplified by nine cycles of PCR. The sequencing libraries were cleaned up using AMPure XP beads (1:1.8 sample vol. to beads vol.; Beckman Coulter). The size distribution of the libraries were determined by Bioanalyzer (Agilent Technologies). The concentration of the libraries was quantified by GenNext NGS Library Quantification Kit (Toyobo Co., Ltd). The ChIPmentation libraries were sequenced using 150 bp paired-end reads on the HiSeq X (Illumina). The sequence reads that were obtained were mapped to the human hg19 genome by bowtie 2 . 


\section{Computational Methods}

Functional analysis of differentially methylated regions

GO analysis of differentially methylated regions was performed using GREAT[53].

Enriched GO lists were summarized based on Semantic Similarity by the GOsemSim R package.

\section{Screening of DNA methylation-regulating transcription factors}

TFBM overrepresentation analysis was performed as previously described with an additional modification. Briefly, sequences located $\pm 5 \mathrm{kbp}$ from the methylated or demethylated probe positions and the same number of randomly selected probes were extracted from version hg19 of the human genome sequence. TFBM identification was performed using the matchPWM command of the Biostrings package of Bioconductor with the PWM database of Integrated analysis of Motif Activity and Gene Expression changes of transcription factors (IMAGE). Out of the overrepresented motifs, the corresponding genes whose CAGE tag per million $\geq 50$ at the time points where the TF binding motif was overrepresented were selected as DNA methylation-regulating transcription factors.

\section{Correlation matrix}

The correlation coefficient of all combinations of two clusters was computed using the M-values. The correlation coefficients were visualized as the correlation matrix heatmap. The clusters were ordered based on hierarchical clustering, which was calculated using the hclust and dist functions of the R stats package with the default settings.

\section{Functional analysis of differentially methylated regions}

Differentially methylated CpGs that were identified as $\triangle \mathrm{M}>2$ and \pm 100 bp extended regions from the differentially methylated CpGs were used as differentially methylated regions. The differentially methylated regions were subjected to GREAT analysis using the submitGreatJob function implemented in the rGREAT R package with background data, which is with the regions extended $\pm 100 \mathrm{bp}$ for all methylation array probes. $\log _{10}$ FDR and ratio between the numbers of hit regions and all differentially 
methylated regions of the Top10 overrepresented GOs (Biological Process) were visualized.

Annotation of differentially methylated regions

Gene promoters were defined as $1 \mathrm{kbp}$ upstream and $200 \mathrm{bp}$ downstream regions of genes in gencode human release version 19. The enhancers used in this study were FANTOM 5 human phase 1 and 2 permissive enhancers. Non-promoter and nonenhancer regions were defined as unannotated regions. The complete overlap between uninherited demethylated CpGs and each regulatory region was counted.

Coverage analysis of GATA6 ChIPmentation and Omni-ATAC-seq

Bigwig Coverage files of CAGE and Omni-ATAC-seq were computed using bam2wig.py. The read coverage was visualized in the range between $\pm 5 \mathrm{kbp}$ from the demethylated CpGs using the EnrichedHeatmap function implemented in the EnrichedHeatmap R package.

\section{List of abbreviations}

TFs, transcription factors; TFBM, transcription factor binding motif; PBS, Phosphatebuffered saline; DE, definitive endoderm; UDRs, uninherited demethylated regions

\section{Declarations}

\section{Ethics approval and consent to participate}

Not applicable.

\section{Consent for publication}

Not applicable.

\section{Competing interests}

The authors declare that they have no competing interests.

\section{Funding}

This work was supported by Grant-in-Aid for Scientific Research (C) (19K08852) to TS from Japan Society for the Promoting Science. This work was also supported by a 
research grant from the Ministry of Education, Culture, Sport, Science and Technology of Japan for the RIKEN Center for Integrative Medical Sciences.

\section{Authors' contributions}

TS participated in the study's design, devised the methodology, performed the statistical analyses, carried out the molecular biology studies, acquired the funding, and drafted the manuscript. SM, EF, MK, YM, YT, JL, HN, SA, and YS carried out the molecular biology experiments. HS helped to draft the manuscript, acquired the funding, and supervised the study. All authors read and approved the final manuscript.

\section{Acknowledgments}

We thank Chung-Chau Hon for useful advice in data analysis. We thank Jing-ru Li for experiment supports. We thank Horoyuki Miyoshi and RIKEN BRC for providing lentivirus plasmids. We are grateful to RIKEN IMS, Laboratory for Comprehensive Genomic Analysis, for the Hiseq 2500 sequencing. 


\section{References}

1. Eferl R, Sibilia M, Hilberg F, Fuchsbichler A, Kufferath I, Guertl B, et al. Functions of c-Jun in liver and heart development. J Cell Biol. The Rockefeller University Press; 1999;145:1049-61. http://www.jcb.org

2. Hilberg F, Aguzzi A, Howells N, Wagner EF. C-Jun is essential for normal mouse development and hepatogenesis. Nature. Nature Publishing Group; 1993;365:179-81. https://www.nature.com/articles/365179a0

3. Tirona RG, Lee W, Leake BF, Lan L Bin, Brimer Cline C, Lamba V, et al. The orphan nuclear receptor HNF4 $\alpha$ determines PXR- and CAR-mediated xenobiotic induction of CYP3A4. Nat Med. Nature Publishing Group; 2003;9:220-4.

http://www.nature.com/naturemedicine

4. Jiang F, Yeo C-W, Lee S-S, Oh M-K, Ghim J-L, Shon J-H, et al. Effect of HNF4 $\alpha$ genetic polymorphism G60D on the pharmacokinetics of CYP2D6 substrate tolterodine in healthy Korean individuals. Pharmacogenet Genomics. 2013;23:175-9.

http://journals.lww.com/01213011-201303000-00009

5. Pontoglio M, Barra J, Hadchouel M, Doyen A, Kress C, Bach JP, et al. Hepatocyte nuclear factor 1 inactivation results in hepatic dysfunction, phenylketonuria, and renal Fanconi syndrome. Cell. Cell Press; 1996;84:575-85.

http://www.cell.com/article/S0092867400810338/fulltext

6. Matsuda K, Kobune Y, Noda C, Ichihara A. Expression of GATA-binding transcription factors in rat hepatocytes. FEBS Lett. FEBS Lett; 1994;353:269-72. https://pubmed.ncbi.nlm.nih.gov/7957872/

7. Watt AJ, Zhao R, Li J, Duncan SA. Development of the mammalian liver and ventral pancreas is dependent on GATA4. BMC Dev Biol. BioMed Central; 2007;7:37. http://bmcdevbiol.biomedcentral.com/articles/10.1186/1471-213X-7-37 8. Zhao R, Watt AJ, Li J, Luebke-Wheeler J, Morrisey EE, Duncan SA. GATA6 Is Essential for Embryonic Development of the Liver but Dispensable for Early Heart Formation. Mol Cell Biol. American Society for Microbiology; 2005;25:2622-31. http://mcb.asm.org/ 
9. Li J, Ning G, Duncan SA. Mammalian hepatocyte differentiation requires the transcription factor HNF-4 $\alpha$. Genes Dev. Cold Spring Harbor Laboratory Press; 2000;14:464-74. www.genesdev.org 10. Koutsourakis M, Langeveld A, Patient R, Beddington R, Grosveld F. The transcription factor GATA6 is essential for early extra-embryonic development. Development. 1999;126:723-32. http://www.ncbi.nlm.nih.gov/pubmed/9895320 11. Morrisey EE, Tang Z, Sigrist K, Lu MM, Jiang F, Ip HS, et al. GATA6 regulates HNF4 and is required for differentiation of visceral endoderm in the mouse embryo. Genes Dev. Cold Spring Harbor Laboratory Press; 1998;12:3579-90. www.genesdev.org

12. Matsunaga E, Gonzalez FJ. Specific Cytosine Demethylations within the First Exons of the Rat CYP2D3 and CYP2D5 Genes Are Associated with Activation of Hepatic Gene Expression during Development. DNA Cell Biol. DNA Cell Biol; 1990;9:443-52. https://pubmed.ncbi.nlm.nih.gov/2206401/

13. Curradi M, Izzo A, Badaracco G, Landsberger N. Molecular Mechanisms of Gene Silencing Mediated by DNA Methylation. Mol Cell Biol. American Society for Microbiology; 2002;22:3157-73./pmc/articles/PMC133775/?report=abstract 14. Guo H, Zhu P, Yan L, Li R, Hu B, Lian Y, et al. The DNA methylation landscape of human early embryos. Nature. Nature Publishing Group; 2014;511:606-10. https://www.nature.com/articles/nature13544

15. Smith ZD, Meissner A. DNA methylation: Roles in mammalian development [Internet]. Nat. Rev. Genet. Nature Publishing Group; 2013. p. 204-20. www.nature.com/reviews/genetics 16. Bird A. DNA methylation patterns and epigenetic memory [Internet]. Genes Dev. Cold Spring Harbor Laboratory Press; 2002. p. 6-21. http://www.genesdev.org/cgi/doi/10.1101/ 17. Hackett JA, Azim Surani M. DNA methylation dynamics during the mammalian life cycle [Internet]. Philos. Trans. R. Soc. B Biol. Sci. Royal Society; 2013. /pmc/articles/PMC3539357/?report=abstract 
18. Tronche F, Rollier A, Bach I, Weiss MC, Yaniv M. The rat albumin promoter: cooperation with upstream elements is required when binding of APF/HNF1 to the proximal element is partially impaired by mutation or bacterial methylation. Mol Cell Biol. American Society for Microbiology; 1989;9:4759-66. http://mcb.asm.org/ 19. Piechocki MP, Burk RD, Ruch RJ. Regulation of connexin32 and connexin43 gene expression by DNA methylation in rat liver cells. Carcinogenesis. Oxford University Press; 1999;20:401-6. https://academic.oup.com/carcin/article/20/3/401/2526607 20. Vieira I, Sonnier M, Cresteil T. Developmental expression of CYP2E1 in the human liver Hypermethylation control of gene expression during the neonatal period. Eur $\mathrm{J}$ Biochem. Blackwell Publishing Ltd.; 1996;238:476-83. https://febs.onlinelibrary.wiley.com/doi/full/10.1111/j.1432-1033.1996.0476z.x 21. Hammons GJ, Yan-Sanders Y, Jin B, Blann E, Kadlubar FF, Lyn-Cook BD. Specific site methylation in the 5'-flanking region of CYP1A2: Interindividual differences in human livers. Life Sci. Pergamon; 2001;69:839-45.

22. Yokomori N, Kobayashi R, Moore R, Sueyoshi T, Negishi M. A DNA methylation site in the male-specific P450 (Cyp 2d-9) promoter and binding of the heteromeric transcription factor GABP. Mol Cell Biol. American Society for Microbiology; 1995;15:5355-62. http://mcb.asm.org/

23. Yokomori N, Nishio K, Aida K, Negishi M. Transcriptional regulation by HNF-4 of the steroid 15a-hydroxylase p450 (Cyp2a-4) gene in mouse liver. J Steroid Biochem Mol Biol. Pergamon; 1997;62:307-14.

24. Kwon MS, Kim SJ, Lee SY, Jeong JH, Lee ES, Kang HS. Epigenetic silencing of the sulfotransferase 1A1 gene by hypermethylation in breast tissue. Oncol Rep. Spandidos Publications; 2006;15:27-32. http://www.spandidospublications.com/10.3892/or.15.1.27/abstract 25. Dannenberg LO, Edenberg HJ. Epigenetics of gene expression in human hepatoma cells: Expression profiling the response to inhibition of DNA methylation and histone deacetylation. BMC Genomics. BioMed Central; 2006;7:181. https://bmcgenomics.biomedcentral.com/articles/10.1186/1471-2164-7-181 
26. Sgodda M, Aurich H, Kleist S, Aurich I, König S, Dollinger MM, et al. Hepatocyte differentiation of mesenchymal stem cells from rat peritoneal adipose tissue in vitro and in vivo. Exp Cell Res. Academic Press Inc.; 2007;313:2875-86.

27. Yoshida Y, Shimomura T, Sakabe T, Ishii K, Gonda K, Matsuoka S, et al. A role of Wnt/ $\beta$-catenin signals in hepatic fate specification of human umbilical cord bloodderived mesenchymal stem cells. Am J Physiol Liver Physiol. American Physiological Society; 2007;293:G1089-98.

https://www.physiology.org/doi/10.1152/ajpgi.00187.2007

28. Stock P, Staege MS, Müller LP, Sgodda M, Völker A, Volkmer I, et al. Hepatocytes Derived From Adult Stem Cells. Transplant Proc. Elsevier; 2008;40:620-3.

29. Hashimoto K, Kouno T, Ikawa T, Hayatsu N, Miyajima Y, Yabukami H, et al. Single-cell transcriptomics reveals expansion of cytotoxic CD4 T cells in supercentenarians. Proc Natl Acad Sci U S A. 2019;116.

30. Xie S, Wang Z, Okano M, Nogami M, Li Y, He WW, et al. Cloning, expression and chromosome locations of the human DNMT3 gene family. Gene. Elsevier; 1999;236:87-95.

31. Jeltsch A. Molecular enzymology of mammalian DNA methyltransferases. Curr Top Microbiol Immunol. Springer, Berlin, Heidelberg; 2006. p. 203-25.

https://link.springer.com/chapter/10.1007/3-540-31390-7_7

32. Kim JK, Samaranayake M, Pradhan S. Epigenetic mechanisms in mammals

[Internet]. Cell. Mol. Life Sci. Springer; 2009. p. 596-612.

/pmc/articles/PMC2780668/?report=abstract

33. Yen R whay C, Vertino PM, Nelkin BD, Yu JJ, El-deiry W, Cumaraswamy A, et al. Isolation and characterization of the cDNA encoding human DNA methyltransferase.

Nucleic Acids Res. Oxford University Press; 1992;20:2287-91.

/pmc/articles/PMC312343/?report=abstract

34. Goll MG, Bestor TH. Eukaryotic cytosine methyltransferases [Internet]. Annu. Rev. Biochem. Annu Rev Biochem; 2005. p. 481-514.

https://pubmed.ncbi.nlm.nih.gov/15952895/ 
35. Ohno R, Nakayama M, Naruse C, Okashita N, Takano O, Tachibana M, et al. A replication-dependent passive mechanism modulates DNA demethylation in mouse primordial germ cells. Dev. Oxford University Press for The Company of Biologists Limited; 2013;140:2892-903. https://dev.biologists.org/content/140/14/2892 36. Tahiliani M, Koh KP, Shen Y, Pastor WA, Bandukwala H, Brudno Y, et al. Conversion of 5-methylcytosine to 5-hydroxymethylcytosine in mammalian DNA by MLL partner TET1. Science (80- ). American Association for the Advancement of Science; 2009;324:930-5.

www.sciencemag.org/cgi/content/full/1169786/DC1VOL324SCIENCEwww.sciencema g.org

37. Ito S, Dalessio AC, Taranova O V., Hong K, Sowers LC, Zhang Y. Role of tet proteins in $5 \mathrm{mC}$ to $5 \mathrm{hmC}$ conversion, ES-cell self-renewal and inner cell mass specification. Nature. Nature Publishing Group; 2010;466:1129-33. https://www.nature.com/articles/nature09303 38. Ito S, Shen L, Dai Q, Wu SC, Collins LB, Swenberg JA, et al. Tet proteins can convert 5-methylcytosine to 5-formylcytosine and 5-carboxylcytosine. Science (80- ). American Association for the Advancement of Science; 2011;333:1300-3. www.sciencemag.org/cgi/content/full/333/6047/1296/DC1

39. Kohli RM, Zhang Y. TET enzymes, TDG and the dynamics of DNA demethylation [Internet]. Nature. Nature Publishing Group; 2013. p. 472-9. https://www.nature.com/articles/nature 12750 40. He YF, Li BZ, Li Z, Liu P, Wang Y, Tang Q, et al. Tet-mediated formation of 5carboxylcytosine and its excision by TDG in mammalian DNA. Science (80- ). American Association for the Advancement of Science; 2011;333:1303-7. www.sciencemag.org/cgi/content/full/science.1210597/DC1 41. Maiti A, Drohat AC. Thymine DNA glycosylase can rapidly excise 5formylcytosine and 5-carboxylcytosine: Potential implications for active demethylation of CpG sites. J Biol Chem. American Society for Biochemistry and Molecular Biology; 2011;286:35334-8. http://www.jbc.org/ 
42. Inoue A, Shen L, Dai Q, He C, Zhang Y. Generation and replication-dependent dilution of $5 \mathrm{fC}$ and $5 \mathrm{caC}$ during mouse preimplantation development. Cell Res. Nature Publishing Group; 2011;21:1670-6. www.cell-research.com

43. Song CX, He C. Potential functional roles of DNA demethylation intermediates [Internet]. Trends Biochem. Sci. NIH Public Access; 2013. p. 480-4. /pmc/articles/PMC4013277/?report=abstract

44. Suzuki T, Shimizu Y, Furuhata E, Maeda S, Kishima M, Nishimura H, et al. RUNX1 regulates site specificity of DNA demethylation by recruitment of DNA demethylation machineries in hematopoietic cells. Blood Adv. 2017;1.

45. Suzuki T, Maeda S, Furuhata E, Shimizu Y, Nishimura H, Kishima M, et al. A screening system to identify transcription factors that induce binding site-directed DNA demethylation. Epigenetics and Chromatin. 2017;10:60.

https://epigeneticsandchromatin.biomedcentral.com/articles/10.1186/s13072-017-01696

46. de la Rica L, Rodríguez-Ubreva J, García M, Islam ABMMK, Urquiza JM, Hernando H, et al. PU.1 target genes undergo Tet2-coupled demethylation and DNMT3b-mediated methylation in monocyte-to-osteoclast differentiation. Genome Biol. BioMed Central; 2013;14:R99. http://genomebiology.biomedcentral.com/articles/10.1186/gb-2013-14-9-r99 47. Costa Y, Ding J, Theunissen TW, Faiola F, Hore TA, Shliaha P V., et al. NANOGdependent function of TET1 and TET2 in establishment of pluripotency. Nature. Nature Publishing Group; 2013;495:370-4. https://www.nature.com/articles/nature11925 48. Guilhamon P, Eskandarpour M, Halai D, Wilson GA, Feber A, Teschendorff AE, et al. Meta-analysis of IDH-mutant cancers identifies EBF1 as an interaction partner for TET2. Nat Commun. Nature Publishing Group; 2013;4:1-9. www.nature.com/naturecommunications 49. Fujiki K, Shinoda A, Kano F, Sato R, Shirahige K, Murata M. PPAR $\gamma$-induced PARylation promotes local DNA demethylation by production of 5- 
hydroxymethylcytosine. Nat Commun. Nature Publishing Group; 2013;4:1-8.

www.nature.com/naturecommunications

50. Sardina JL, Collombet S, Tian T V., Gómez A, Di Stefano B, Berenguer C, et al. Transcription Factors Drive Tet2-Mediated Enhancer Demethylation to Reprogram Cell Fate. Cell Stem Cell. Cell Press; 2018;23:727-741.e9.

51. Murata M, Nishiyori-Sueki H, Kojima-Ishiyama M, Carninci P, Hayashizaki Y, Itoh M. Detecting expressed genes using CAGE. Methods Mol Biol. Humana Press Inc.; 2014;1164:67-85. https://link.springer.com/protocol/10.1007/978-1-4939-0805-9_7 52. Hay DC, Zhao D, Fletcher J, Hewitt ZA, McLean D, Urruticoechea-Uriguen A, et al. Efficient Differentiation of Hepatocytes from Human Embryonic Stem Cells Exhibiting Markers Recapitulating Liver Development In Vivo. Stem Cells. Wiley; 2008;26:894-902. http://doi.wiley.com/10.1634/stemcells.2007-0718

53. McLean CY, Bristor D, Hiller M, Clarke SL, Schaar BT, Lowe CB, et al. GREAT improves functional interpretation of cis-regulatory regions. Nat Biotechnol. 2010;28:495-501.

54. Yu G, Li F, Qin Y, Bo X, Wu Y, Wang S. GOSemSim: An R package for measuring semantic similarity among GO terms and gene products. Bioinformatics. Oxford Academic; 2010;26:976-8. http://bioconductor.org/packages/2.6/bioc/html/GOSemSim.html 55. Madsen JGS, Rauch A, Van Hauwaert EL, Schmidt SF, Winnefeld M, Mandrup S. Integrated analysis of motif activity and gene expression changes of transcription factors. Genome Res. Cold Spring Harbor Laboratory Press; 2018;28:243-55. http://www.genome.org/cgi/doi/10.1101/gr.227231.117.

56. Fuest M, Willim K, MacNelly S, Fellner N, Resch GP, Blum HE, et al. The transcription factor c-Jun protects against sustained hepatic endoplasmic reticulum stress thereby promoting hepatocyte survival. Hepatology. John Wiley \& Sons, Ltd; 2012;55:408-18. http://doi.wiley.com/10.1002/hep.24699 57. Hasselblatt P, Rath M, Komnenovic V, Zatloukal K, Wagner EF. Hepatocyte survival in acute hepatitis is due to c-Jun/AP-1-dependent expression of inducible nitric 
oxide synthase. Proc Natl Acad Sci U S A. National Academy of Sciences; 2007;104:17105-10. www.pnas.org/cgi/content/full/

58. Behrens A, Sibilia M, David JP, Möhle-Steinlein U, Tronche F, Schütz G, et al. Impaired postnatal hepatocyte proliferation and liver regeneration in mice lacking c-jun in the liver. EMBO J. John Wiley \& Sons, Ltd; 2002;21:1782-90.

https://www.embopress.org/doi/full/10.1093/emboj/21.7.1782

59. Schmidl C, Rendeiro AF, Sheffield NC, Bock C. ChIPmentation: Fast, robust, lowinput ChIP-seq for histones and transcription factors. Nat Methods. Nature Publishing Group; 2015;12:963-5. http://chipmentation.computational-epigenetics.org/.

60. Andersson R, Sandelin A. Determinants of enhancer and promoter activities of regulatory elements [Internet]. Nat. Rev. Genet. Nature Research; 2020. p. 71-87. www.nature.com/nrg

61. Corces MR, Trevino AE, Hamilton EG, Greenside PG, Sinnott-Armstrong NA, Vesuna S, et al. An improved ATAC-seq protocol reduces background and enables interrogation of frozen tissues. Nat Methods. Nature Publishing Group; 2017;14:95962. https://www.nature.com/articles/nmeth.4396

62. Thakur A, Wong JCH, Wang EY, Lotto J, Kim D, Cheng J, et al. Hepatocyte Nuclear Factor 4-Alpha Is Essential for the Active Epigenetic State at Enhancers in Mouse Liver. Hepatology. John Wiley and Sons Inc.; 2019;70:1360-76. https://onlinelibrary.wiley.com/doi/abs/10.1002/hep.30631

63. Merika M, Orkin SH. DNA-binding specificity of GATA family transcription factors. Mol Cell Biol. American Society for Microbiology; 1993;13:3999-4010. /pmc/articles/PMC359949/?report=abstract 64. Vakoc CR, Letting DL, Gheldof N, Sawado T, Bender MA, Groudine M, et al. Proximity among distant regulatory elements at the $\beta$-globin locus requires GATA-1 and FOG-1. Mol Cell. Elsevier; 2005;17:453-62. http://www.molecule.org/cgi/content/full/17/3/453/ 
65. Fisher JB, Pulakanti K, Rao S, Duncan SA. GATA6 is essential for endoderm formation from human pluripotent stem cells. Biol Open. Company of Biologists Ltd; 2017;6:1084-95. http://bio.biologists.org/

66. Cirillo LA, Lin FR, Cuesta I, Friedman D, Jarnik M, Zaret KS. Opening of compacted chromatin by early developmental transcription factors HNF3 (FoxA) and GATA-4. Mol Cell. Elsevier; 2002;9:279-89.

http://www.cell.com/article/S1097276502004598/fulltext

67. Zaret K. Developmental competence of the gut endoderm: Genetic potentiation by GATA and HNF3/fork head proteins. Dev. Biol. Academic Press Inc.; 1999. p. 1-10. 68. Zaret KS, Watts J, Xu J, Wandzioch E, Smale ST, Sekiya T. Pioneer factors, genetic competence, and inductive signaling: Programming liver and pancreas progenitors from the endoderm. Cold Spring Harb Symp Quant Biol. Cold Spring Harbor Laboratory Press; 2008. p. 119-26. http://symposium.cshlp.org/content/73/119

69. Mayran A, Drouin J. Pioneer transcription factors shape the epigenetic landscape [Internet]. J. Biol. Chem. American Society for Biochemistry and Molecular Biology Inc.; 2018. p. 13795-804. http://www.jbc.org/

70. Galas DJ, Schmitz A. DNAase footprinting a simple method for the detection of protein-DNA binding specificity. Nucleic Acids Res. Oxford Academic; 1978;5:315770. https://academic.oup.com/nar/article/5/9/3157/2380868

71. Kodo K, Nishizawa T, Furutani M, Arai S, Yamamura E, Joo K, et al. GATA6 mutations cause human cardiac outflow tract defects by disrupting semaphorin-plexin signaling. Proc Natl Acad Sci U S A. National Academy of Sciences; 2009;106:139338. http://genome.lbl.gov/vista/

72. De Franco E, Shaw-Smith C, Flanagan SE, Shepherd MH, Hattersley AT, Ellard S. GATA6 mutations cause a broad phenotypic spectrum of diabetes from pancreatic agenesis to adult-onset diabetes without exocrine insufficiency. Diabetes. American Diabetes Association; 2013;62:993-7. /pmc/articles/PMC3581234/?report=abstract 
73. Allen HL, Flanagan SE, Shaw-Smith C, De Franco E, Akerman I, Caswell R, et al. GATA6 haploinsufficiency causes pancreatic agenesis in humans. Nat Genet. Nature Publishing Group; 2012;44:20-2. http://www.nature.com/naturegenetics/. 